\title{
Ankündigungen
}

\section{Calls for Papers}

Gender, Bodies and Technology, Interdisciplinary Conference Women's and Gender Studies Program at Virginia Tech, 22.-24. April 2010, Roanoke, VA Einsendeschluss: 15. September 2009

Weitere Informationen: http://www.cpe.vt.edu/gbt/

Reusing the Industrial Past, Joint Conference 2010 ICOHTEC und TICCIH, 10.-15. August 2010, Tampere, Finnland

Einsendeschluss: 16. November 2009

Weitere Informationen: http://www.tampere.fi/industrialpast2010

\section{Tagungen}

2.-3. Juli 2009, Society for the Study of Nineteenth-century Ireland: Science and Technology in Nineteenth-century Ireland, Dublin

Weitere Informationen: http://www.ssnci.com/conferences

3.-4. Juli 2009, Nachwuchstagung: Knowledge Has Left the Building. LaborWissen - Transformation, Ruhr-Universität Bochum

Weitere Informationen: http://labor-wissen-transformation.de

3.-4. Juli 2009, Centre for Birmingham and Midlands History: ,Where Genius and the Arts Preside'. Matthew Boulton and the Soho Manufactory 18092009, Birmingham, UK

Weitere Informationen: http://www.cbamh.bham.ac.uk/

9.-11. Juli 2009, University of York, Cultural History Conference 2009: Cultural Histories of Sociability, Spaces and Mobility, York, UK

Weitere Informationen: http://www.york.ac.uk/depts/hist/research/conf/ sociability.html

16.-17. Juli 2009, National Maritime Museum: The Long View: 400 Years of the Telescope, Greenwich, UK

Weitere Informationen: http://www.nmm.ac.uk/researchers/conferences-andseminars/thelongview

28. Juli - 2. August 2009, XXIII International Congress of History of Science and Technology (ICOHTEC und IUHPS Joint Conference): Ideas and Instruments in Social Context, Budapest

Weitere Informationen: http://www.conferences.hu/ichs09/ 
5.-7. August 2009, IEEE Conference: History of Technical Societies, Philadelphia, PA

Weitere Informationen: http://www.ieee.org/go/historyconference

30. August - 5. September 2009, XIV. International TICCIH Congress: Industrial Heritage, Ecology and Economy, Freiberg

Weitere Informationen: http://www.ticcih2009.de/

1.-4. September 2009, CRESC 5th Annual Conference 2009: Objects - What Matters? Technology, Value and Social Change, Manchester

Weitere Informationen: http:/www.cresc.ac.uk/events/conference2009/

4.-5. September 2009, Workshop University of St Andrews: Engineering Space in the Nineteenth Century: Environment, Science, Technology and the Transformation of Space, St Andrews, UK

Weitere Informationen: http://www.st-andrews.ac.uk/history/

20.-22. September 2009, 14th Artefacts Meeting: The Relations of Science and Technology as Portrayed in Museums, Science Museum, London

24.-26. September 2009, 3rd International Conference International Railway History Association/Association Internationale d'Histoire des Chemins de fer/Associacion Internacional de Historia Ferroviaria: Railways in Transition. Eastern Europe Railways: Past, Present and Future in the 20th and 21st Centuries, Bratislava

Weitere Informationen: http://www.aihc-irha-aihf.com

24.-27. September 2009, 92. Jahrestagung der Deutschen Gesellschaft für Geschichte der Medizin, Naturwissenschaft und Technik e.V. und der Gesellschaft für Wissenschaftsgeschichte: Symposium on Knowledge and Ideology, Hannover

Weitere Informationen: http://www.dggmnt.de/

\section{Preise}

Paul-Bunge-Preis 2010 der Hans R. Jenemann-Stiftung, getragen von der Gesellschaft Deutscher Chemiker (GDCh) und der Deutschen Bunsen-Gesellschaft für Physikalische Chemie (DBG)

Bewerbungsschluss: 30.9.2009

Weitere Informationen: http://www.gdch.de/gdch/eps/ausschr/bunge07.htm

Habilitationspreise 2010 und Hedwig-Hintze-Preis 2010 des Verbands der Historiker und Historikerinnen Deutschlands

Bewerbungsschluss: 31. Oktober 2009

Weitere Informationen: http://www.vhd.gwdg.de/preise.html 


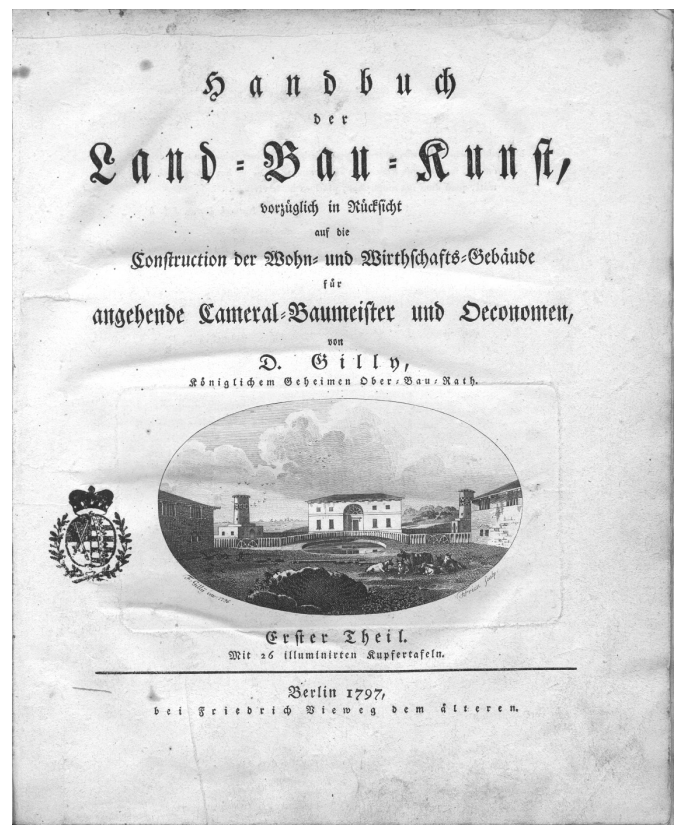

\section{Umschlagbild}

Im Jahr 1797 veröffentlichte der preußische Landbaumeister David Gilly das Handbuch der Land-Bau-Kunst, das bis in die 1830er Jahre mehrere Auflagen erlebte. Bislang vor allem als eines der bedeutendsten Werke des frühen Klassizismus in Deutschland wahrgenommen, das den ländlichen Baugeschmack nachhaltig beeinflusste, richtet sich aus der Perspektive der Umweltgeschichte das Augenmerk auf das innovative Moment von Gillys Forderung nach dauerhaftem und sparsamem, insbesondere holzsparendem Bauen. Die eingehende Thematisierung der Baumaterialien und die Aufforderung zur Exploration mineralischer Ressourcen stellten im Sinne der ökonomischen Aufklärung einen wichtigen Beitrag für die Modernisierung des ländlichen Raums dar.

Quelle: Titelblatt des ersten Bandes des Handbuchs der Land-Bau-Kunst (1797). SLUB Dresden/Deutsche Fotothek/Archit. 689-1 (Titelblatt) 